\title{
A Thermal Analysis Method for Fuel-Cooled Combustor Structure based on Heat Flux Measurement
}

\author{
Yang $\mathrm{Lu}^{1}$, Long $\mathrm{Li}^{2}$, Xuejun $\mathrm{Fan}^{3}$ \\ LHD, Institute of Mechanics, Chinese Academy of Sciences, Beijing, 100190, P. R. China
}

\begin{abstract}
In this paper, a heat flux measurement based thermal analysis method has been developed to analysize the heat transfer process within fuel-cooled structure of a combustor. By using data obtained from newly-upgraded heat flux sensors developed based on the principle of Gardon heat-flux gauge, the complex heat transfer processes relevant to such cooling structure exposed in severe heat environment are able to be simplified to two coupled process: flow and convective heat transfer of fuel within cooling channels and heat transfer within cooling channels-embeded combustor structure. The validness and overall correctness of this analysis method are tested using data of a Mach 2.5 supersonic combustor. The comparation between the simulation and experiment results are satisfactory. The relatively simpliness, decent accuracy and low computation cost make it promising to put this analysis method into engineering use.
\end{abstract}

\section{Nomenclature}

$A \quad=$ area

$S \quad=$ perimeter

$\dot{q} \quad=$ heat flux

$\overline{\dot{q}} \quad=$ average heat flux

$\rho \quad=$ density

$h \quad=$ enthalpy

htc $\quad=$ heat transfer coefficient

$T_{w} \quad=$ temperature of wall

$T_{f} \quad=$ temperature of fuel

$\tau_{w} \quad=$ friction of wall

$C_{f} \quad=$ friction coefficient

$S_{t} \quad=\quad$ Stanton number

$N_{u} \quad=\quad$ Nusselt number

$P_{r} \quad=$ Prandtl number

\section{Introduction}

$\mathrm{R}$ egenerative cooling using onboard fuel has been proposed ${ }^{1}$ and demonstrated ${ }^{2}$ to be a reliable thermal management solution for practical scramjet engines. The heat transfer processes related to a regenerative cooling system are very complicated, which could be divided into three coupled parts: the heat from combustion and aerodynamic heating transferred to the wall, the heat conducted through the three-dimensional engine structure, and the heat absorbed by fuel via a convective heat transfer. In order to aid in designing and optimizing actively cooling

\footnotetext{
${ }^{1}$ Assistant Professor, National Key Laboratory of High Temperature Gas Dynamics, luyang@imech.ac.cn.

${ }^{2}$ Post doctor, National Key Laboratory of High Temperature Gas Dynamics, 1 li918@126.com.

${ }^{3}$ Professor, National Key Laboratory of High Temperature Gas Dynamics, xfan@imech.ac.cn, Member AIAA.
} 
system, substantial efforts in developing analyzing tools or CFD simulation have been made. Traci et al. ${ }^{3}$ developed a systematical thermal analysis code called VITMAC for active cooling in RBCC system. Bouchez et al. ${ }^{4}$ considered fuel cracking and developed a code named NANCY for active cooling analysis in dual-mode ramjets. Gamble et al. ${ }^{5}$ brought a new heat exchanger analysis tool, SRHEAT, for scramjet/ramjet active cooling use. Recently, Cho et al. ${ }^{6}$ reported a design program for regenerative cooling liquid rocket engine.

However, the heat transfer processes related to the regenerative cooling system are too complicated. On one hand, turbulent mixing, combustion and the near-wall heat transfer process predicted using many existing CFD tools are still lack high accuracy or rather time-consuming. On the other hand, the fuel temperature varies significantly in the cooling processes and its state changes from liquid state to supercritical state or even to partially cracked. Consequently, the thermo-physical and transport properties of the fuel change dramatically, which will affect the heat transfer between the fuel and structure. However, many tools lack the validation of some important assumptions or empirical formula they have used. As a result, further studies for fuel-cooled combustor structure analysis methods are still in need.

A heat flux experimental data based thermal analytical tool was developed in this research to handle regenerative cooling thermal analysis difficulties. The new heat flux sensors are designed based on the principle of Gardon heat flux meter. By using a bypass heat flow path with active cooling, the measurement range of this type of sensor is extended greatly from approximately $0.1 \mathrm{MW} / \mathrm{m}^{2}$ to nearly $400 \mathrm{~W} / \mathrm{cm}^{2}$. Aditionally, by carefully adjusting the heat resistances of the two heat flow paths, a good sensitivity and time response of less than 10 seconds are achieved. With this heat flux measurement method developed recently by authors' group, thermal environment of "hot wall" side of the active cooling structure can be known with high accuracy and this difficult process can be decoupled from the whole active-cooling process. So the overall problem is reduced to only two rather than three coupled processes. Accordingly, the thermal analytical tool consists of two main modules: the analysis module of fuel flowing and heat convection within cooling channels and the analysis module of heat transfer within cooling structure. Each of them can function individually or together to meet different design requirements. When these two modules are iteratively used together with heat flux measurement data, a coupled thermal analysis computation method for actively cooling combustor structure is achieved with moderate calculation cost and decent accuracy compared to the results of experimental measurements.

\section{Overview of analysis method and iteration process}

Figure.1 gives a schematic drawing of a cross section of fuel-cooled structure for analysis. Subscript 1 represents variables of combustor inner hot wall. Subscript 2 represents variables of the cooling channel wall. Subscript 3 represents variables of combustor outside cold wall. $\mathrm{h}$ in figure. 1 and 2 denotes heat transfer coefficient.

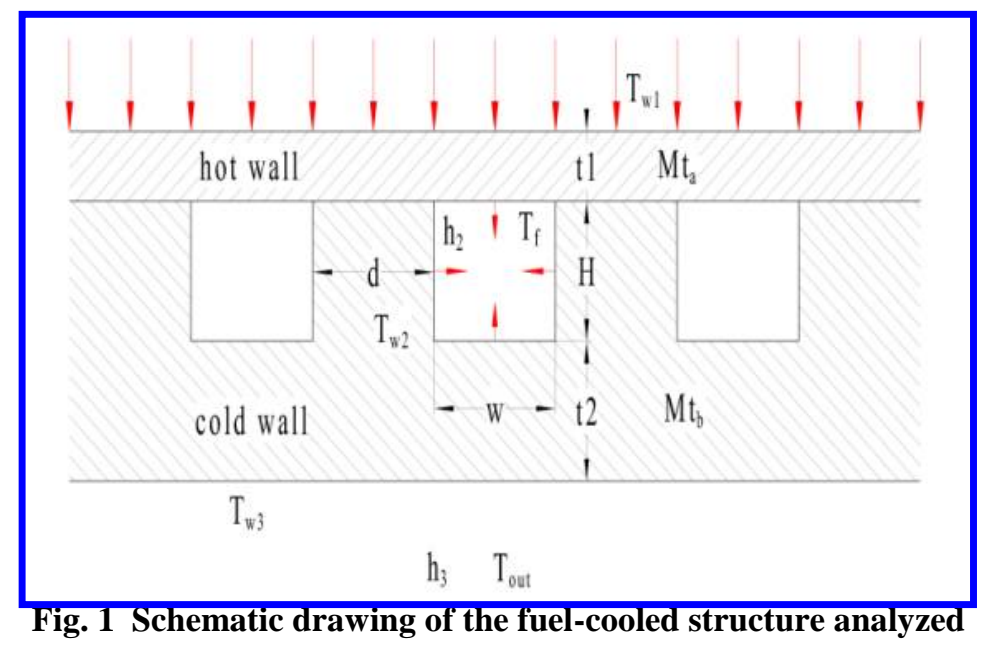

Firstly, heat flux measurement is performed and after treatment inner hot wall heat flux $\dot{q}_{w 1}$ distribution along flow direction can be known. Secondly, with assumed $T_{w 2}$ distribution, the cooling channels analysis module is invoked and it can provide distributions of convection coefficient $h t c_{2}$ and coolant fuel temperature $T_{f}$ along flow 
direction. After that, heat conduction analysis module is invoked within every cross section of cooling structure and structure temperature distribution can be obtained. Then, $T_{w 2}$ distribution can be updated and used for next cycle of the cooling channels and heat conduction analysis. Iteration is carried on until convergence is reached. The whole computational process is shown in Figure 2. Details of each module will be given below.

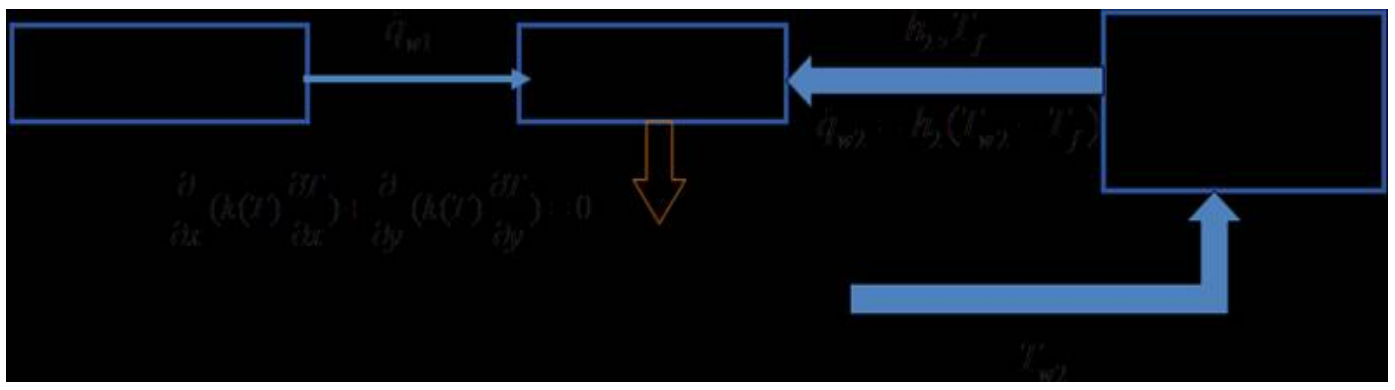

Fig. 2 Relation structure of computation modules and iteration process

\section{Detailed description of the analysis method}

\section{A. Heat flux measurement and inner hot wall boundary condition treatment}

One key feature of this research, the inner hot wall boundary condition specification, is based on heat flux measurement progress made recently in authors' group. Li et. al ${ }^{7}$ has developed a reliable heat flux measurement method for high temperature environment. The heat flux sensor is designed based on the principle of Gardon heat flux gauge. Due to the use of recirculating cooling water and heat bypass, the measurement range of the sensor has been extended significantly. Consequently, measurements of high levels of heat flux can be realized with credibility. Figure. 3 is a schematic of the upgraded heat flux sensor. Additional information for this heat flux sensor can be found in reference 7 .

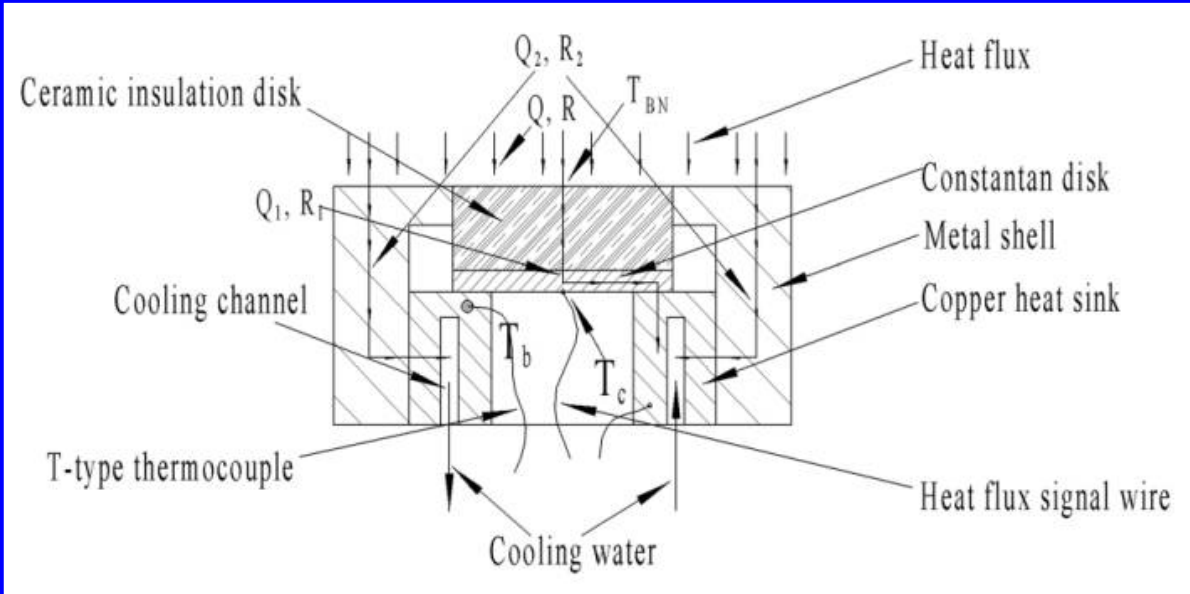

Fig. 2 Schematic of the upgraded heat flux sensor(from Ref. 7)

To calibrate the sensor at high temperature and heat flux, which are expected to be encountered in typical highMach combustors, a blackbody cavity calibration system has been designed and fabricated, in which a blackbody cavity can be heated up to $2500^{\circ} \mathrm{C}$ and corresponds to a heat flux of $350 \mathrm{~W} / \mathrm{cm}^{2}$. 


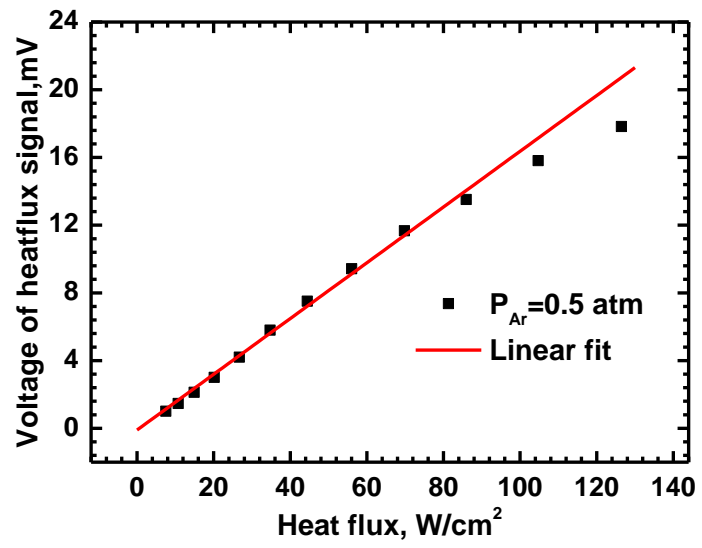

Fig. 4 Blackbody calibration curve for the heat flux sensor. (from Ref. 7)

Figure 4 shows the resulting calibration curve for such a heat flux sensor. Linear dependence of the sensor's output voltage on the applied heat flux can be observed for the radiation heat flux from zero to less than approximately $120 \mathrm{~W} / \mathrm{cm}^{2}$. These heat flux sensors have been successfully utilized in a water-cooled supersonic model combustor (Fig. 5) and measurements were carried out in this supersonic model combustor with liquid kerosene injection into a Mach 2.5 vitiated air flow of total temperatures ranging from 1200 to $1900 \mathrm{~K}$ and total pressure of around 1.4 $\mathrm{MPa}$. The combustor was cooled with recirculating water to make the experiment time longer enough for the whole system to reach thermal equilibrium. Seven heat flux sensors were installed along the centerline of the ton combustor wall in the flow direction as shown.

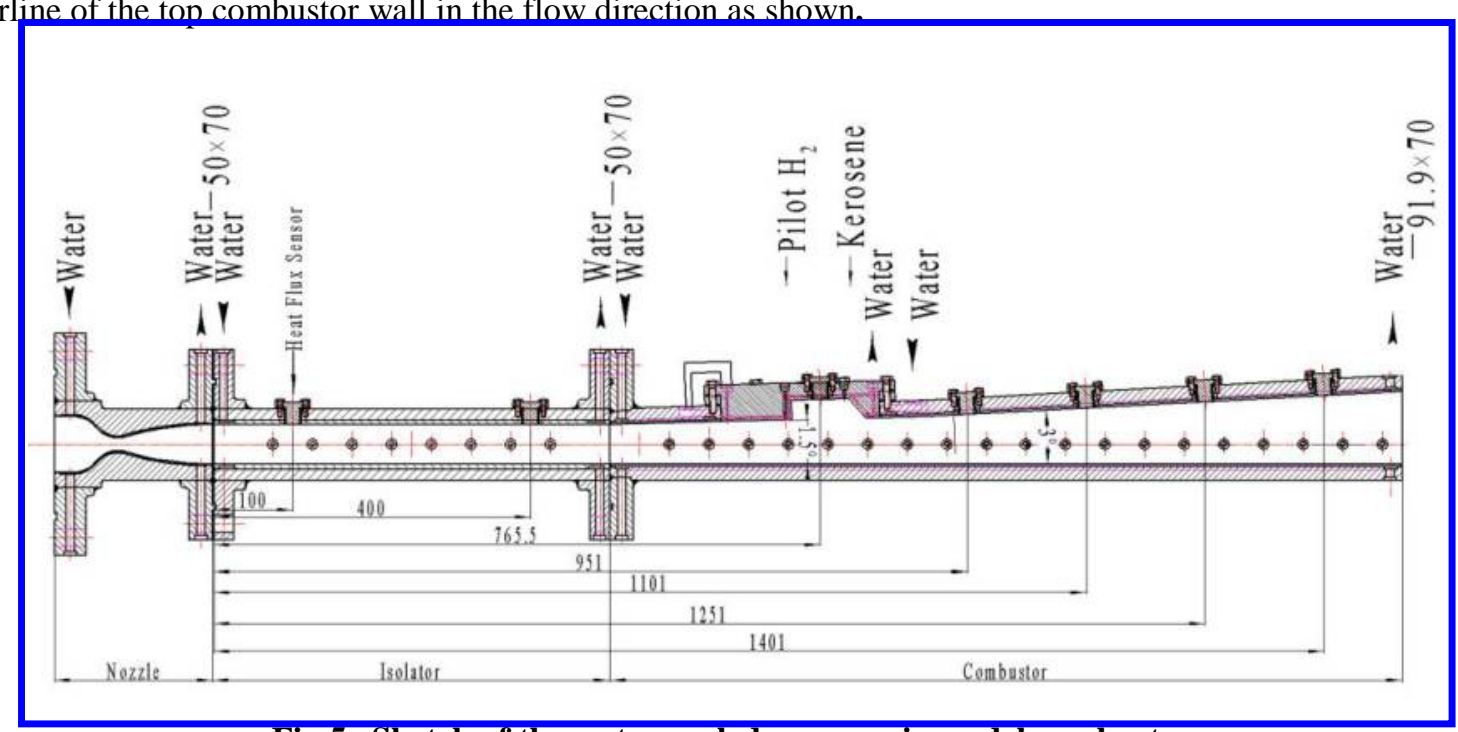

Fig.5 Sketch of the water cooled supersonic model combustor.

Most of the sensors installed survived after 50 experimental runs. The outputs for same test conditions displayed good repeatability. Typical measured heat flux distributions with and without combustion along the combustor flow direction is shown in Figure 6. 


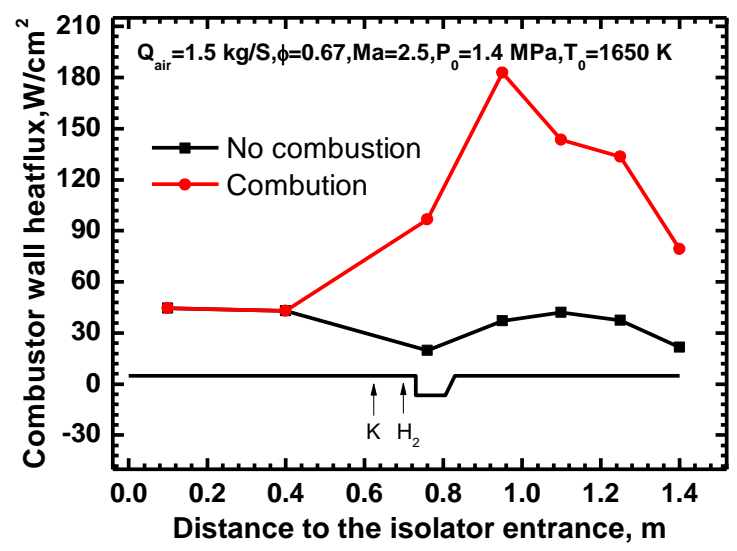

Fig.6 Heat flux distributions along the combustor with and without combustion.

Inlet and outlet coolant water temperatures and mass flow rate were measured and recorded. With known geometry information, the average heat flux distribution along a cooling panel can be calculated:

$$
\overline{\dot{q}_{w 1, w a t e r}}=\frac{\dot{m} \cdot\left(h_{\text {out }}\left(T_{\text {out }}\right)-h_{\text {in }}\left(T_{\text {in }}\right)\right)}{A_{\text {panel }}}
$$

Furthermore, another almost same model combustor is manufactured with the only difference is that it was no longer water-cooled but fuel-cooled. Inlet and outlet coolant fuel temperatures and pressures as well as mass flow rate were measured and recorded for use. A lab-developed 3-species surrogate fuel model is employed for SUPERTRAPP ${ }^{8}$ use to estimate the enthalpy $h_{\text {out }}\left(p_{\text {out }}, T_{\text {out }}\right), h_{\text {in }}\left(p_{\text {in }}, T_{\text {in }}\right)$ of coolant fuel. So, the average heat flux of the fuel-cooled panel can be calculated:

$$
\overline{\dot{q}_{w 1, f u e l}}=\frac{\dot{m} \cdot\left(h_{\text {out }}\left(p_{\text {out }}, T_{\text {out }}\right)-h_{\text {in }}\left(p_{\text {in }}, T_{\text {in }}\right)\right)}{A_{\text {panel }}}
$$

Because the fuel-cooled and water-cooled combustors are nearly the same except for the coolant, it is reasonable to assume that under same working conditions, the heat flux distributions along flow direction of these two combustors are of very similar "shape" but different absolute values. An absolute value difference factor (DF) defined below can be introduced and considered to be constant along flow direction within two combustors:

$$
D F=\frac{\overline{\dot{q}_{w 1, \text { fuel }}}}{\overline{\dot{q}_{w 1, \text { water }}}}
$$

With DF known, the similar law of heat flux distribution can be established: the one-dimensional heat flux distribution of fuel-cooled combustor under the same working condition is predictable by the measured water-cooled heat flux counterpart:

$$
\dot{q}_{w 1, \text { fuel }}(x)=D F \cdot \dot{q}_{w 1, \text { water }}(x)
$$

Now the hot wall boundary condition is obtained and ready to be used for structure heat conduction analysis.

\section{B. Flow and convective heat transfer of fuel within cooling channels}

Considering the fact that practically-used cooling channels are usually long and narrow, convective heat transfer process of fuel in such cooling channels can be approximated to be one dimensional with decent accuracy. Figure 7 shows the control volume for analysis: 


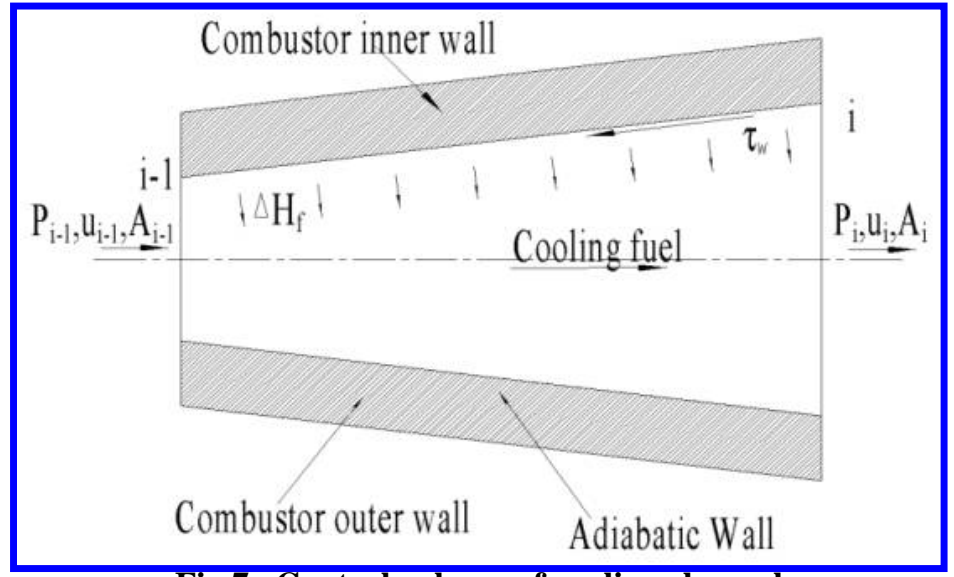

Fig.7 Control volume of cooling channel

For the governing equations we have:

$$
\begin{gathered}
\dot{m}_{f}=\rho_{i} u_{i} A_{i}=\rho_{i-1} u_{i-1} A_{i-1} \\
\dot{m}_{f} u_{i-1}+P_{i-1} A_{i-1}-\int_{i-1}^{i} P d A=\dot{m}_{f} u_{i-1}+P_{i} A_{i}+\int_{i-1}^{i} \tau_{w} d A_{f} \\
\dot{m}_{f}\left(h_{i-1}+\frac{u_{i-1}^{2}}{2}\right)+\Delta H_{f, i-1}=\dot{m}_{f}\left(h_{i}+\frac{u_{i}^{2}}{2}\right)
\end{gathered}
$$

$\dot{m}$ is the mass flow rate of the coolant fuel in a cooling channel. $\Delta H_{f, i-1}$ denotes the heat absorbed by coolant from the wall of the cooling channel and its formula is:

$$
\Delta H_{f}=\dot{q}_{w, f} S_{f} \Delta x, \dot{q}_{w, f}=h t c_{f}\left(T_{w 2}-T_{f}\right), h t c_{f}=\frac{N u_{f} \lambda_{w}}{D_{e h}}
$$

$S_{f}$ is the local perimeter of the cooling channel cross section and $D_{e h}$ is the hydraulic diameter of the cooling channel. Distributions of cooling channel wall temperature $-T_{w 2}$, which are assumed in this module, and the heat transfer coefficient of cooling channel wall - $h t c_{f}$ will be used in structure analysis later. It is not a flaw to assume cooling channel wall temperature's distribution here because it will be updated each time after the heat transfer analysis of cooling structure (discussed in the next section) until convergence is achieved.

The whole cooling channel is divided into lots of subsections to insure the difference of properties of two adjacent sections is small and the following approximations can be used:

$$
\int_{i-1}^{i} P d A \approx \frac{P_{i}+P_{i-1}}{2}\left(A_{i}-A_{i-1}\right) \int_{i-1}^{i} \tau_{w} d A_{c h} \approx \frac{\tau_{w, i-1} S_{f, i-1}+\tau_{w, i} S_{f, i}}{2} \Delta x
$$

By definition for Newton fluid, we have :

$$
\tau_{w}=\frac{C_{f}}{2} \rho u^{2}
$$

And Reynold analogy is used to get friction coefficient:

$$
\frac{C_{f}}{2}=S t \operatorname{Pr}^{\frac{2}{3}} S t=\frac{N u}{\operatorname{Re} \operatorname{Pr}}
$$

The merit of doing this is that not only friction effect is considered but also pressure drop in cooling channels is no need to be estimated by using empirical formulas in after-calculation analysis as most previous studies have done, but rather are calculated during iteration and coupled within the governing equations. It adds the coherence of the whole analysis method.

As mentioned in the introduction, there is no justification for arbitrarily using formulas developed for water or any other matter other than hydrocarbon fuel to describe coolant heat transfer in regenerative cooling system. 
Suitable formulas are required for describing hydrocarbon fuel heat convection process. These formulas should be directly experimentally obtained with hydrocarbon fuel as working substance or at least are accepted to be applicable to such coolant substance, such as Gnielinski formula ${ }^{9}$ :

$$
N u=0.012\left(\operatorname{Re}_{f}^{0.87}-280\right) \operatorname{Pr}^{0.4}\left(\operatorname{Pr}_{f} / \operatorname{Pr}_{w}\right)^{0.11}, 1.5 \leq \operatorname{Pr}_{f} \leq 500,3000<\operatorname{Re}_{f}<10^{6}
$$

With all abovementioned equations and formulas, local velocity of fuel can be obtained iteratively. Fuel temperature and other important physical variables, which are considered functions of local fuel temperatures and pressures, can also be deduced. It should be emphasized that functions of SUPERTRAPP code are used again during computation to obtain these state and flow variables since hydrocarbon fuels are more complicated than water:

$$
h_{f}=h_{f}(P, T), \ldots, \rho_{f}=\rho_{f}(P, T)
$$

\section{Heat transfer analysis within cooling structure}

Considering that the cooling panel usually has high aspect ratio and the temperature gradient in flow direction is smaller than that in the cross section plane, the problem can be reduced to a collection of two-dimension heat conduction reasonably. Moreover, benefitting from this treatment, this module can be conveniently programmed in parallel, which turned out to be a very efficient method to perform on multi-core multi-threads desktops.

It is noteworthy that many previous researches treated the problem by simply analyzing only one channel within the structure with periodic boundary condition. However, as shown in this study this approximation is questionable and not proper for heat analysis for rectangular cross section cooling structure.

The controlling equation for two-dimensional heat conduction is:

$$
\rho c \frac{\partial T}{\partial t}=\frac{1}{\partial x}\left(\lambda \frac{\partial T}{\partial x}\right)+\frac{\partial}{\partial y}\left(\lambda \frac{\partial T}{\partial y}\right)+S
$$

A standard practice to solve this equation is to use finite volume method. However, when multiple channels and lots of cross sections along flow direction are involved, the cost of computation can be fairly large. So in this research, a thermo-resistance formula is employed and only steady state situation is considered and an algebra expression ${ }^{10}$ for $\mathrm{Ti}, \mathrm{j}$ can be used (take non-boundary node expression for example):

$$
\begin{gathered}
T_{i, j}=\frac{\frac{T_{i+1, j}}{R_{1}}+\frac{T_{i, j-1}}{R_{2}}+\frac{T_{i-1, j}}{R_{3}}+\frac{T_{i, j+1}}{R_{4}}}{\frac{1}{R_{1}}+\frac{1}{R_{2}}+\frac{1}{R_{3}}+\frac{1}{R_{4}}} \\
\text { With }\left\{\begin{array}{l}
R_{1}=\frac{\Delta X_{i+1, j}}{\Delta Y_{i+1, j}} \frac{1}{2}\left(\frac{1}{K_{i, j}}+\frac{1}{K_{i+1, j}}\right), R_{2}=\frac{\Delta Y_{i, j-1}}{\Delta X_{i, j-1}} \frac{1}{2}\left(\frac{1}{K_{i, j}}+\frac{1}{K_{i, j-1}}\right) \\
R_{3}=\frac{\Delta X_{i-1, j}}{\Delta Y_{i-1, j}} \frac{1}{2}\left(\frac{1}{K_{i, j}}+\frac{1}{K_{i-1, j}}\right), R_{4}=\frac{\Delta Y_{i, j+1}}{\Delta X_{i, j+1}} \frac{1}{2}\left(\frac{1}{K_{i, j}}+\frac{1}{K_{i, j+1}}\right)
\end{array}\right.
\end{gathered}
$$

$\mathrm{Ri}(\mathrm{i}=1 \ldots)$ denotes thermo-resistance from a specific direction. $\mathrm{Ki}, \mathrm{j}$ denotes thermal conductivity coefficient, which varies with temperature as $K(T)=A T+B$ in a wide range. A and B are material-related constants and can be retrieved from material database. For rectangular cross-section channels in rectangular coordinates (as shown in Fig.1):

$$
\begin{gathered}
\Delta X_{i+1, j}=\Delta X_{i-1, j}=\Delta X_{i, j+1}=\Delta X_{i, j-1}=\Delta x \\
\Delta Y_{i+1, j}=\Delta Y_{i-1, j}=\Delta Y_{i, j+1}=\Delta Y_{i, j-1}=\Delta y
\end{gathered}
$$

Free convection or adiabatic boundary conditions are applied to all walls except the inner hot wall in that regenerative cooling system is exposed in the open air. The hot wall boundary condition is obtained by measurement and treatment discussed in section A. The coolant fuel temperature and heat transfer coefficient are calculated from analysis of convective heat transfer of fuel in section B. Now, boundary conditions specifications are completed and heat transfer process of cooling structure can be analyzed.

American Institute of Aeronautics and Astronautics 


\section{Results and discussion}

The analysis method is verified by comparing computational results to experiment results obtained under the same working conditions. The kerosene-cooled supersonic combustion experiment parameters are: $\mathrm{Qt}=1.47 \mathrm{Kg} / \mathrm{s}$, $\mathrm{Pt}=1.38 \mathrm{MPa}, \mathrm{Tt}=1500 \mathrm{~K}$ and the mass flow rate of coolant for the cooling plate analyzed is $52.2 \mathrm{~g} / \mathrm{s}$.

Figure 8-10 are pictures of calculated two-dimensional temperature distributions of cooling structure at different locations along flow direction using method discussed in part III. It can be found out from these figures that it is very likely the hottest part of the cooling structure lies in the two corners adjacent to the inner combustor wall. This three dimensional effect is easy to be missing, which may cause dangerous design problems.

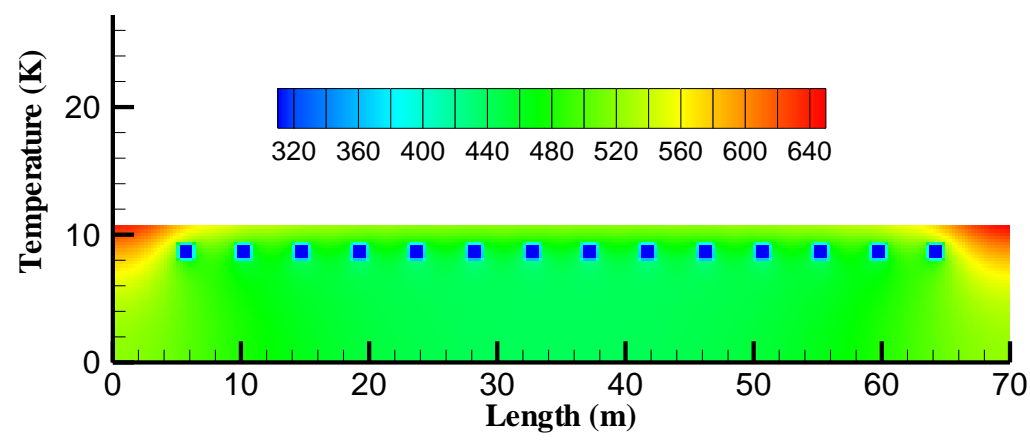

Fig.8 Calculated temperature distribution of entrance cross section

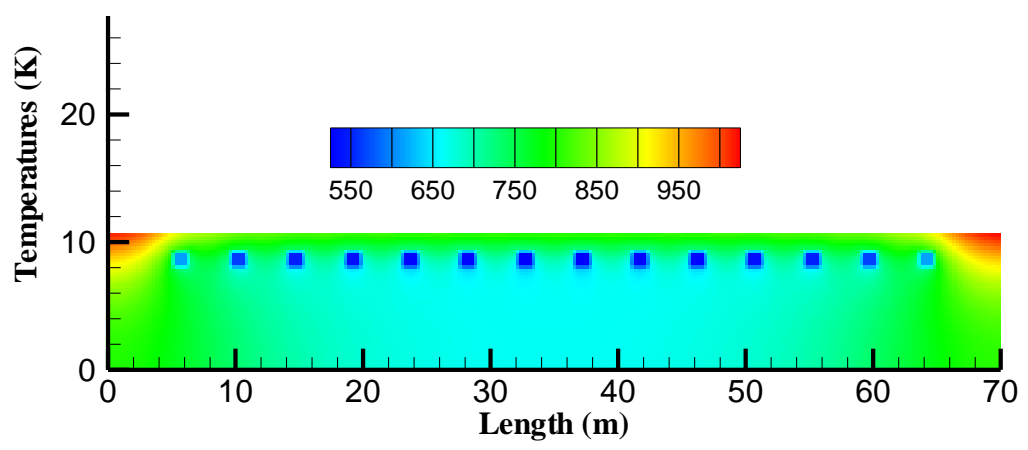

Fig.9 Calculated temperature distribution of a middle cross section

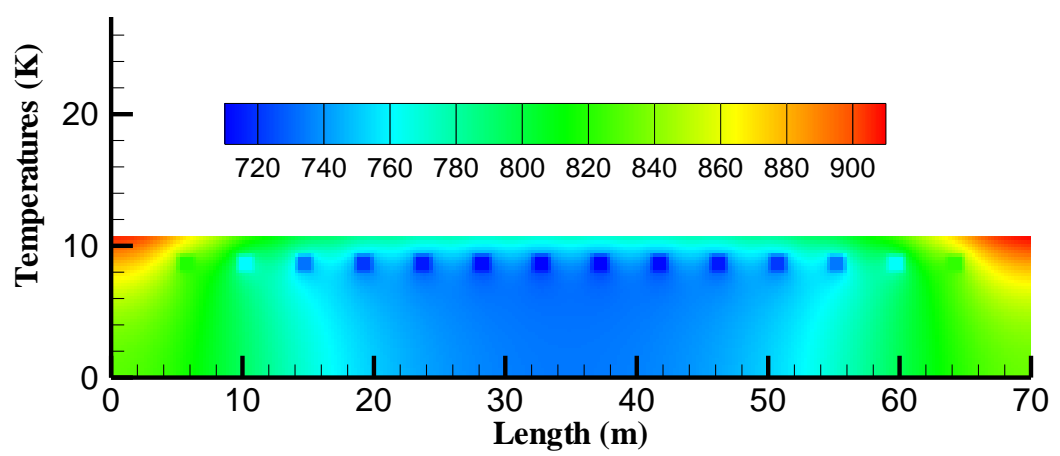

Fig.10 Calculated temperature distribution of exit cross section

With data of every cross section assembled together, the whole three-dimensional temperature distribution of the fuel-cooled combustor structure can be known. 
Figure 11 compares the calculated averaged outside wall temperature distribution and averaged cooling fuel temperature distribution with those measured in the experiment.

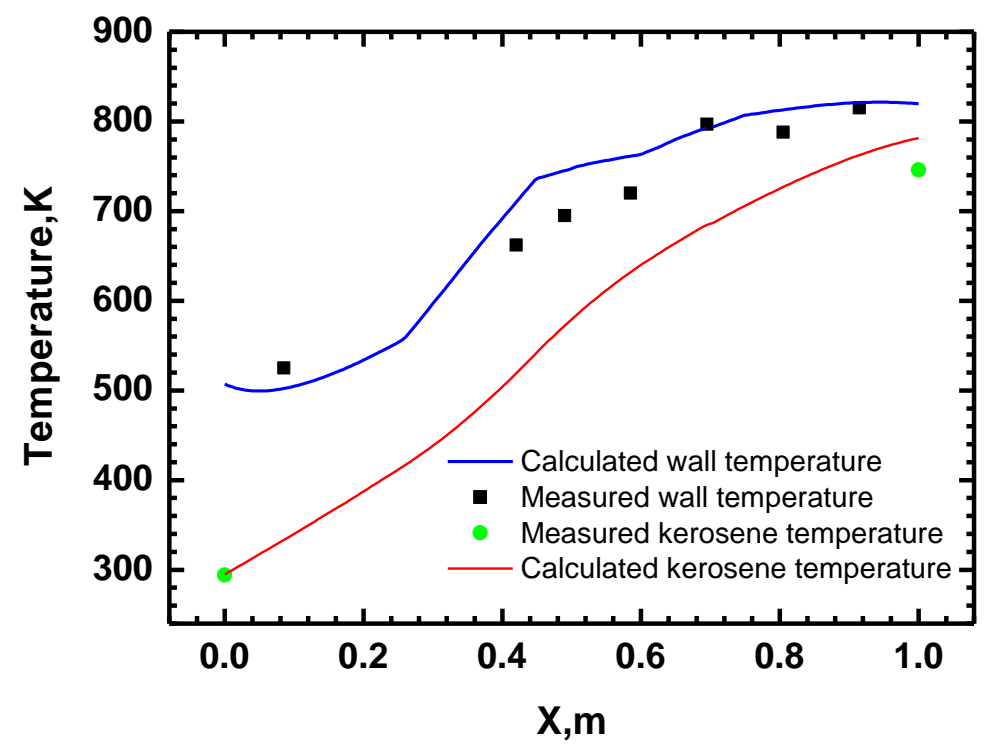

Fig.11 Temperature distributions of kerosene and outside wall (calculated and measured)

It can be told from Fig.11 that the simulation results are rather close to those experimental results. Temperature distributions calculated are generally slightly higher than measurements. We attributed these differences to the following possible two reason: (1) in the experiment, the apparatus exposed in the open air so that the convection of wall with surroundings as well as radiation heat loss is inevitable but in the computational analysis, these effects are neglected. (2) the calculated temperature displayed in figure 11 is an averaged one along the outside wall line perpendicular to the flow direction while the measured temperature is obtained by thermocouples placed in the middle of the outside wall line. As can be seen from figure 8-10, the central area temperature of the outside wall is always a little smaller than the two ends.

\section{Conclusion and future work}

As presented previously, this newly developed heat analysis method based upon heat flux measurement is valid and able to capture some three-dimensional effect of fuel-cooled supersonic combustor structure. Besides, the computation cost is relatively low. So this method is quite promising in engineering practice.

In the future, mildly-cracked model for hydrocarbon fuel can be introduced into the analysis of convective heat transfer of fuel in cooling channels in order to describe the behavior of partially- cracked coolant. More work should be done to develop an advanced surrogate fuel model and to find better formulas required for describing hydrocarbon fuel heat convection process.

\section{Acknowledgments}

Current research program at the LHD, CAS was supported by the National Natural Science Foundation of China under Contractor No. 91016005, 10621202 and LHD Youth Innovative Foundation. The authors would like to acknowledge Mr Linjin Meng, Mr Peng Huang, Mr Jinquan Wang and Mr. Y. Li for their technical support.

\section{References}

${ }^{1}$ Wagner, W.R. and Shoji, J.M., “Advanced Regenerative Cooling Techniques for Future Space Transportation Systems," AIAA-75-1247, 1975.

${ }^{2}$ Powell, O.A., Edwards, J.T., Norries, R.B., Numbers, K.E. and Pearce, J.A., "Development of Hydrocarbon-Fueled Scramjet Engines: The Hypersonic Technology(HyTech) Program,” Journal of Propulsion and Power, Vol. 17, No. 6, 2001, pp. 1170-1176. 
${ }^{3}$ M.Traci R, L.Farr J, Jr., et al., “A Thermal Management Systems Model for the NASA GTX RBCC Concept,” NASA/CR2002-211587, 2002.

${ }^{4}$ Bouchez M, Dufour E, Daniau E., "Semi-empirical and CFD Analysis of Actively Cooled Dual-mode Ramjets:2006 status," AIAA 2006-8073, 2006.

${ }^{5}$ Gamble E J, Gutierrez J, Giet D, et al., "Development of a Scramjet/Ramjet Heat Exchanger Analysis Tool," AIAA 20084614, 2008.

${ }^{6}$ Won Kook Cho, Woo Seok Seol, Min Son et al., "Development of Prelimiary Design Program for Combustor of Regenerative Cooled Liquid Rocket Engine," Journal of Thermal Science, Vol. 20, No. 5, 2011, pp. 467-473.

${ }^{7}$ Long Li, Xuejun Fan, et al., "Measurements of Wall Heat Flux and Temperature in a supersonic Model Combustor," AIAA249406-323, 2011.

${ }^{8}$ Ely J, Huber M., "NIST standard reference database 4-NIST thermo physical properties of hydrocarbon mixtures," National Inst. of Standard, Gaithersburg, MD, 1990.

${ }^{9}$ V. Gnielinski., "New equations for heat and mass transfer in turbulent pipe and channel flow," Internatinal Chemical Engineering, Vol. 16, No. 2, 1976, pp. 359-367.

${ }^{10}$ Earl A. Thornton., "A Finite Element Program for Steady-state Thermal Analysis of Convectively Cooled Strutures," NASA/CR-145069,1976. 